\title{
Discrete self-similarity in the formation of satellites for viscous cavity break-up
}

\author{
Marco A. Fontelos and Qiming Wang
}

(Dated: December 11, 2020)

\begin{abstract}
The breakup of a jet of a viscous fluid with viscosity $\mu_{1}$ immersed into another viscous fluid with viscosity $\mu_{2}$ is considered in the limit when the viscosity ratio $\lambda=\mu_{1} / \mu_{2}$ is close to zero. We show that, in this limit, a transition from ordinary continuous self-similarity to discrete self-similarity takes place as $\lambda$ decreases. It follows that instead of a single point breakup, the rupture of the inner jet occurs through the appearance of an infinite sequence of filaments of decreasing size that will eventually produce infinite sequences of bubbles of the inner fluid inside the outer fluid. The transition can be understood as the result of a Hopf bifurcation in the system of equations modelling the physical problem.
\end{abstract}

\section{INTRODUCTION}

The breakup of fluid filaments into drops is one of the most relevant and widely studied problems in fluid mechanics. It is both a fundamental problem since the times of Savart, Plateau and Rayleigh [1], 2], 3] and an issue of paramount importance for technological aplications based on inkjet printing technology, micro and nano-encapsulation, production of filaments by electrospinning, etc (see 44 for a general review). The complementary problem, i.e. the closure of an axisymmetric cavity and the formation of bubbles, has also been widely studied due to its important role as a mechanism for air entrainment into the ocean [5] and also connected to technical applications [6]. When a viscous thread or a cavity breaks-up into drops, it is frequently the case that the result is a sequence of drops or bubbles of similar size surrounded by droplets/bubbles of much smaller size called satellite drops/bubbles [7], [8]. The origin and possible control of such satellites remains a puzzle and an obstacle for many practical applications where precise knowledge of the size of the drops/bubbles produced is needed. Remarkably, the pinch-off of a liquid thread is a singularity [9] governed by simple similarity laws and occurs according to selfsimilar functions as first described in [10]. In this letter we describe and explain the formation of generations of satellites in the breakup of a very low viscosity fluid jet inside a viscous environment as the result of a discretely self-similar (DSS) process. This is in contrast with continuous self-similarity that explains the disconnection of a single drop from a jet: DSS is a periodic process in similarity variables, i.e. a process that repeats itself in a logarithmic time scale (as one approaches a singular time) and decreasing spatial windows, with the result of infinite sequences of structures of decreasing size [11].

\section{BOUNDARY INTEGRAL EQUATIONS AND SINGULARITY FORMATION}

We consider a viscous jet, with viscosity $\mu_{1}$, inside a viscous surrounding media with viscosity $\mu_{2}$, and define the viscosity contrast parameter $\lambda=\frac{\mu_{1}}{\mu_{2}}$. The fluid variables velocity $\mathbf{v}$ and pressure $p$ for both fluids satisfy Stokes equations with the condition that the difference of viscous stresses at the interface balance surface tension (proportional to the interface's mean curvature $\kappa$, with surface tension coefficient $\gamma$ and along the normal direction $\mathbf{n})$ :

$$
\left(\sigma_{2}-\sigma_{1}\right) \cdot \mathbf{n}=\gamma \kappa \mathbf{n}
$$

where $\sigma_{i}$ is the viscous stress exerted by the fluid $i$ on the interface. The fluid interface, described by the distance $h(z, t)$ to the axis of symmetry, evolves following the velocity field. The interfacial velocities are solved via boundary-integral equations in axisymmetric geometry. The for-aft symmetry is imposed so that only half of the drop shape needs to be determined. The interface velocities are assumed to vary linearly along each boundary element and integral is computed by using standard 4or 6-point Gauss-Legendre formula. The weakly singular integral is solved by using Gauss-log quadrature. After obtaining velocities, the interface is updated by the first order Euler time scheme (details can also be found in [12], [13]). When the drop is close to pinching, the local grid points are redistributed to maintain a smoothly varying spacing proportional to $\arctan \left(\sqrt{\left(z-z_{\text {min }}\right)^{2}+h_{\text {min }}^{2}}\right)$ 14]. The simulation is stopped when the drop radius is about $10^{-4}$ or smaller (when drop interface oscillation occurs, the simulation may stop earlier). Validations of the code are presented as supplementary material.

Direct scaling yields a linear flow for the filament radius vs time near pinch-off and a also a linear axial scale vs time: $h(z, t)=\left(t_{0}-t\right) \varphi\left(\left(z-z_{0}\right) /\left(t_{0}-t\right)\right)$ where $t_{0}, z_{0}$ are the pinchoff time and point respectively and $\varphi(\xi)$ is a function behaving linearly at infinity so that the geometry at pinchoff is that of two cones touching at their vertex (see figure 1). This is the classical result reported in the literature [15], [16], 17]. Nevertheless, as we can appreciate in figures 1, 2, for sufficiently small $\lambda$, corresponding to an inner fluid of very low viscosity, the geometry near pinch-off is not as simple as two cones. The evolution of $h_{\text {min }}$, the minimum jet radius, clearly deviates to the simple linear law and presents a complex 

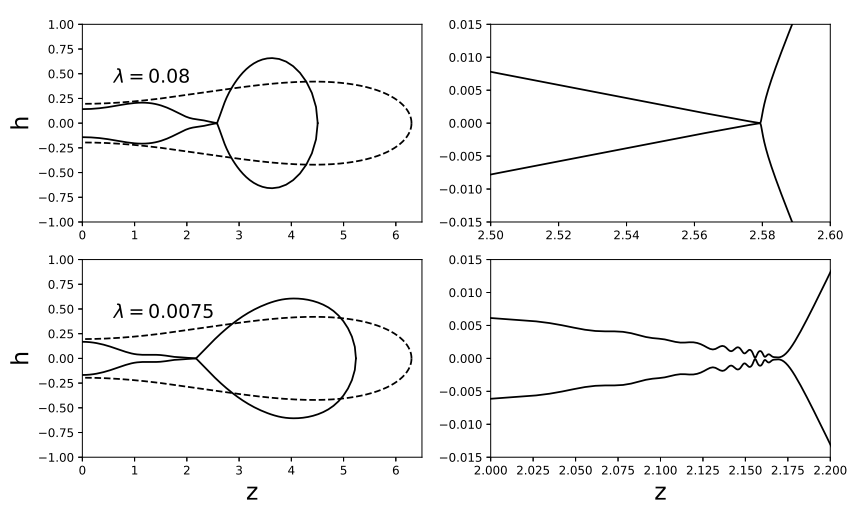

FIG. 1. Evolution of a liquid jet and disconnection of a drop. Dotted line: initial data. Continuous line: finite time breakup through the formation of conical singularities for $\lambda=0.08$ and the formation of a sequence of satellites for $\lambda=$ 0.0075 . The right panels are the zoom-in versions of the left panels, at breakup time $\tilde{t} \approx 6.065$ and $\tilde{t} \approx 2.025$, respectively. Here $\tilde{t}$ is the dimensionless time variable in boundary integral simulation, before rescaling to long wave variable.

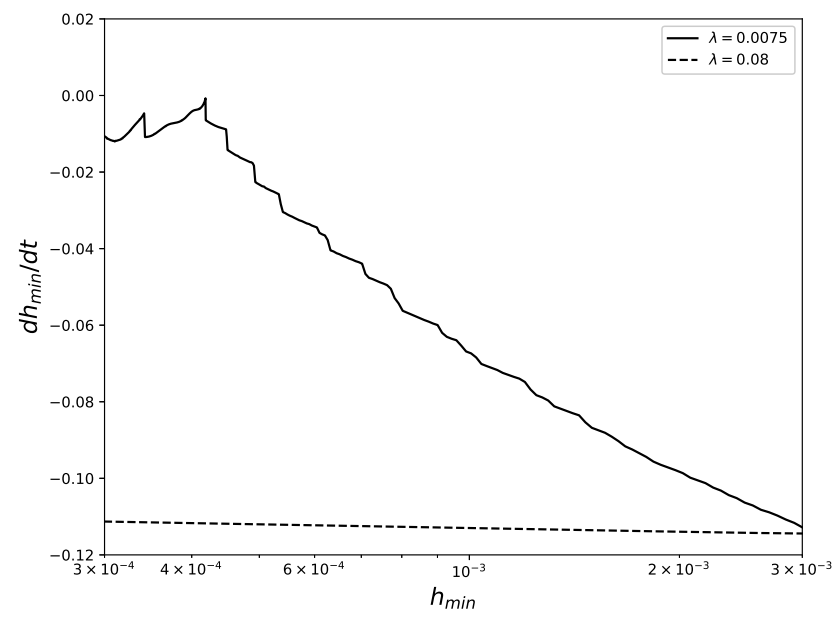

FIG. 2. Evolution of $\frac{d h_{\min }}{d t}$, the velocity of the thinning filament radius vs the minimum radius. According to simple scaling $\frac{d h_{\min }}{d t}$ should converge to a constant ad $h_{\text {min }}$ tends to zero. This is clear for $\lambda=0.08$, but unclear for $\lambda=0.0075$. In the later case, oscillations near pinch-off are observed.

oscillatory behaviour instead. This oscillatory behaviour translates into both the interface and velocity profiles, as seen in figure 3 and results in the formation of sequences of satellites. This is a fact reported in recent experiments [18] in connection with the healing of an annular viscous film.
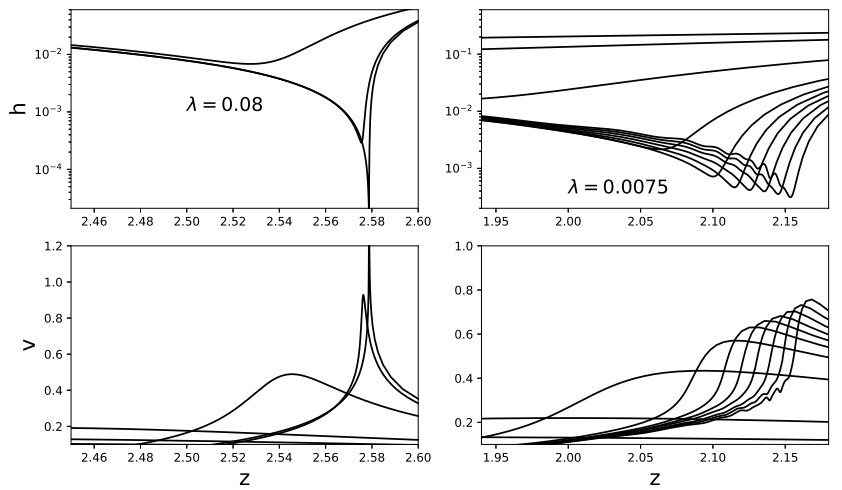

FIG. 3. Profiles and axial velocities of the liquid jet for various times close to the breakup time and showing oscillations in the case $\lambda=0.0075$. The profiles for $\lambda=0.08$ correspond to $\tilde{t}=6.005,6.062,6.065$, while for $\lambda=0.0075$, $\tilde{t}=1.973,1.992,2.00,2.007,2.013,2.018,2.023$.

\section{ASYMPTOTIC MODEL AND DISCRETE SELF-SIMILARITY}

In what follows we study an asymptotic model for the limit of very low viscosity inner fluid inside an outer viscous fluid. More precisely, by considering the viscosity ratio $\lambda=\varepsilon^{2} \lambda_{0}$ where $\lambda_{0}=O(1)$ and $\varepsilon$ is the slenderness ratio between typical transversal and longitudinal length scales. One can then obtain the system (see [19], [15], [13]):

$$
\begin{aligned}
\frac{\partial h}{\partial t} & =\frac{1}{16 \lambda_{0}} \frac{1}{h} \frac{\partial}{\partial z}\left(h^{4} \frac{\partial p}{\partial z}\right) \\
p & =\frac{1}{h}+\frac{2}{h} \frac{\partial h}{\partial t}
\end{aligned}
$$

Note that the change of variables $t \rightarrow 2 t, z \rightarrow z / \sqrt{8 \lambda_{0}}$ allows to remove the constants $\frac{1}{16 \lambda_{0}}$ and 2 from the equations and, after writing from (2)

$$
\frac{\partial h}{\partial t}=(p h-1)
$$

and inserting into (1) we obtain the equation

$$
-\frac{1}{h^{2}} \frac{\partial}{\partial z}\left(h^{4} \frac{\partial p}{\partial z}\right)+p=\frac{1}{h}
$$

The system (3), (4) consists then of an ordinary differential equation for $h$ coupled with a linear elliptic partial differential equation for $p$. We can, in principle, solve the linear problem (4) for the pressure $p$ and write

$$
p=K_{h}\left[\frac{1}{h}\right]
$$

for some nonlinear integral operator $K_{h}$, and insert into (3) to obtain

$$
\frac{\partial h}{\partial t}=\left(K_{h}\left[\frac{1}{h}\right] h-1\right)
$$




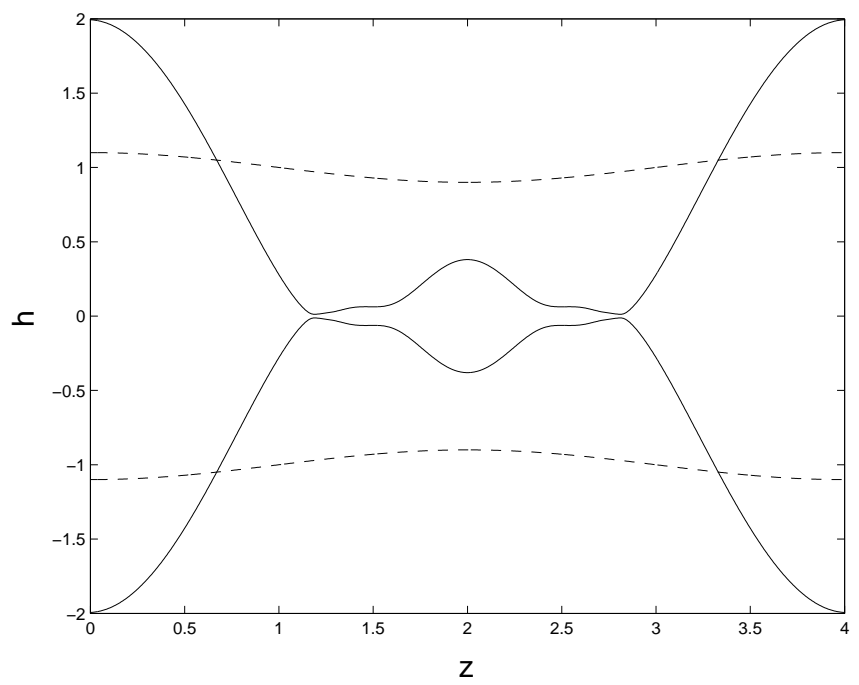

FIG. 4. Evolution of a liquid jet. Dotted line: initial data. Continuous line: finite time breakup through the formation of conical singularities.

which in an integro-differential equation for $h(z, t)$. We will see that, despite its simplicity (a single first order integro-differential PDE), the solutions to equation (5) display a very interesting dynamics directly related to discretely selfsimilar features (see [11]) leading, in this case, to the formation of cascades of satellite bubbles of the inner fluid inside the outer fluid.

We have solved (3), (44) numerically for an initial data $h(z, 0)=1+0.1 \cos (2 \pi z / L)$ with $L=4$ and obtained the profile in figure 4 where finite time breakup develops in the apparent form of two conical shapes touching at the breakup point. This fact can also be observed in figure 7 where we represent the evolution profiles for several times close to the breakup time. The appearance of a seemingly conical break-up is reinforced by the existence of a selfsimilar solution to (3), (4) in the form

$$
\begin{aligned}
& h(z, t)=\left(t_{0}-t\right) H\left(z /\left(t_{0}-t\right)\right) \\
& p(z, t)=\left(t_{0}-t\right)^{-1} P\left(z /\left(t_{0}-t\right)\right)
\end{aligned}
$$

where $t_{0}$ is the breakup time and $H(\xi), P(\xi)$ satisfy the system

$$
\begin{aligned}
-H+\xi H^{\prime} & =H P-1 \\
-\frac{1}{H^{2}}\left(H^{4} P^{\prime}\right)^{\prime}+P & =\frac{1}{H}
\end{aligned}
$$

together with the boundary conditions $H \sim \nu_{ \pm} \xi$, $P \sim \mu_{ \pm} \xi^{-1}$ as $\xi \rightarrow \pm \infty$. Note that the linear asymptotic behaviour of $H$ implies that the local shape near the breakup point is in the form of two cones with semi angles $\theta_{ \pm}=\arctan \nu_{ \pm}$. By means of a Newton iteration scheme we have solved (6), (77) subject to boundary conditions implying a linear asymptotic behaviour of $H(\xi)$ and found the profile represented in dotted lines in figure 6. In particular, we can compute $\theta_{+} \simeq 44^{\circ}$

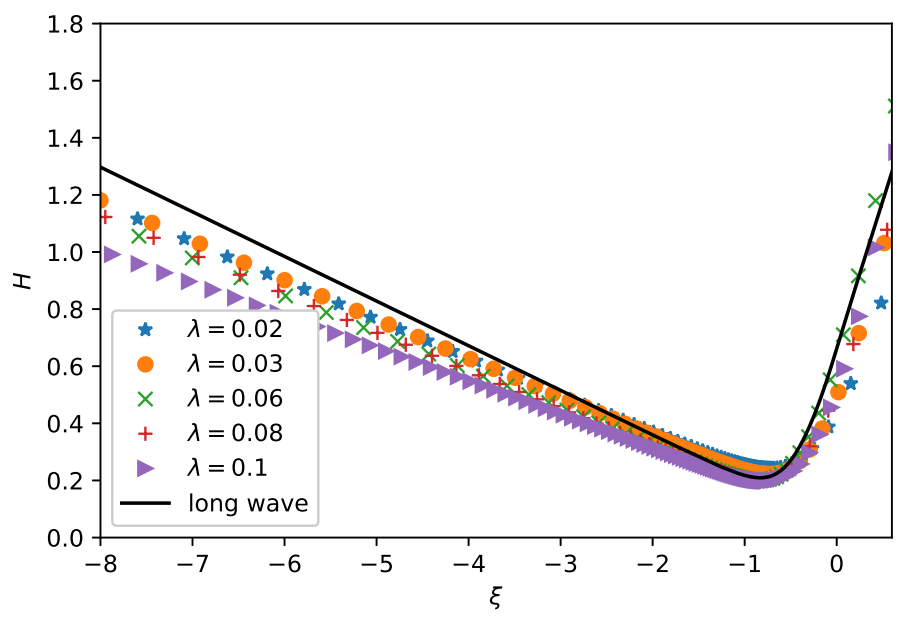

FIG. 5. Comparison between selfsimilar neck profiles for various values of $\lambda$, suitably rescaled, and the selfsimilar solution to the long wave model. Denoting $\tilde{z}$ and $\tilde{h}$ the dimensionless variables in full problem, they are connected to the long wave variables as follows: $\xi=2\left(\tilde{z}-\tilde{z}_{0}\right)\left(\left(\tilde{t}_{0}-\tilde{t}\right) \sqrt{8 \lambda}\right)^{-1}-b \ln \left(\tilde{t}_{0}-\tilde{t}\right)$ and $H=2 \tilde{h}\left(\tilde{t}_{0}-\tilde{t}\right)^{-1}$, where $\tilde{z}_{0}$ and $\tilde{t}_{0}$ are pinch-off location and time, respectively, in boundary integral simulation. The constant $b$ is fitted from simulation.

and $\theta_{-} \simeq 9^{\circ}$. It is worth noting the remarkable good agreement between the selfsimilar solution to the long wave model and the selfsimilar profiles obtained from the full boundary integral calculation (figure 5). In order to compare them we rescale time by a factor of 2 and $z$ by a factor $\sqrt{8 \lambda}=\sqrt{8 \lambda_{0}} \varepsilon$ also see caption in Fig. [5, in agreement with the rescaling that led to (3), (4). We can observe in figure 5 that, very interestingly, the resulting profiles seem to converge towards a universal profile as $\lambda$ decreases and that profile is very close to the selfimilar solution to the long wave model. Nevertheless, as we mentioned above, the comparison cannot hold for artibrarily small values of $\lambda$ due to the development of instabities around the pinch-off point. Below we will present a detailed study of this instabilities in the context of the long wave model.

In figure 7 we plot the same profiles as in figure 6 rescaled by the factor $\left(t_{0}-t\right)^{-1}$ and compared to the selfsimilar solution. As we can see, the numerical profiles evolve around the selfsimilar solution but do not seem to converge to it. In fact, as we approach the singularity, instabilities develop in the form of oscillations whose length-scale decreases as we approach the singularity point (see the undulations in figure 7). This is the signature of discrete self-similarity (DSS), i.e. $\tau$-periodic solutions of the equations (3), (4) in similarity variables 


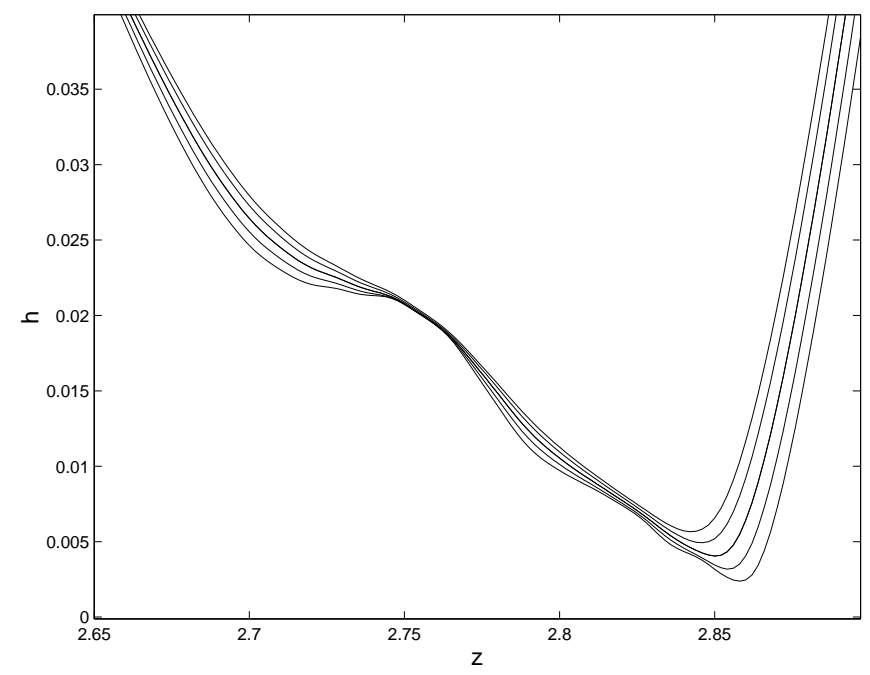

FIG. 6. Numerical profiles for five different times as we approach the singularity. The singularity time is $t_{0}=3.970$ and the profiles correspond to $t=3.940,3.945,3.950,3.955,3.960$.

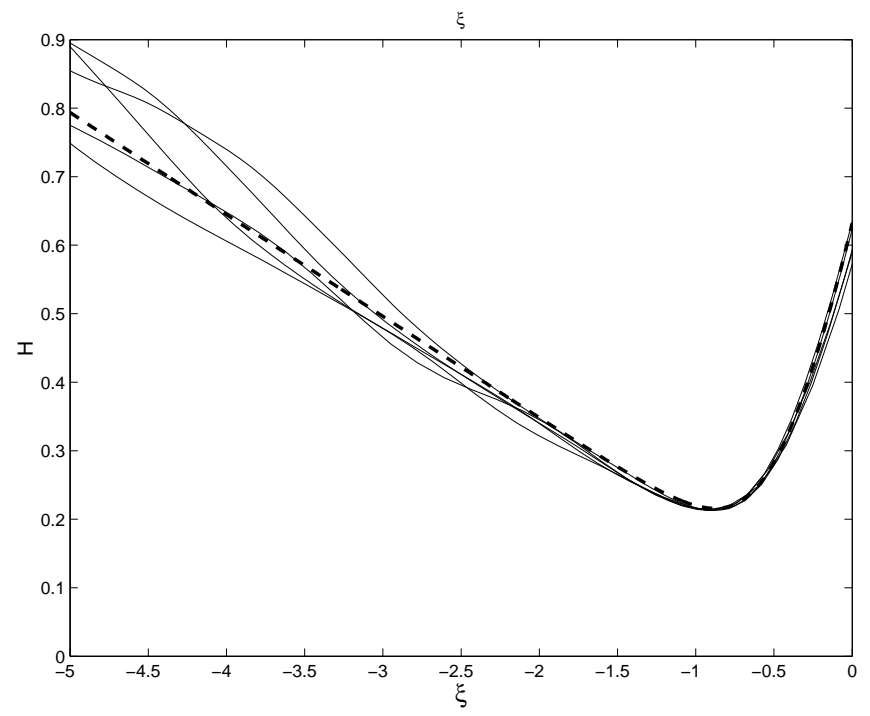

FIG. 7. The profiles of 6 rescaled according to the similarity laws together with the selfsimilar profile.

$$
\begin{aligned}
\tau=-\log \left(t_{0}-t\right), \xi=z /\left(t_{0}-t\right) & : \\
H_{\tau}-H+\xi H^{\prime} & =H P-1 \\
-\frac{1}{H^{2}}\left(H^{4} P^{\prime}\right)^{\prime}+P & =\frac{1}{H}
\end{aligned}
$$

As we showed in [11], discretely self-similar solutions may appear as the result of a Hopf bifurcation around a continuously selfsimilar solution where the later solution becomes unstable giving rise to a periodic orbit around it (the DSS solution). In the present case, our numerical results show evidence of periodicity in the self-similar variables and the result is an infinite sequence of iterated filaments of decreasing length-scale that develop as we approach the singularity (see figure 8). The breakup of

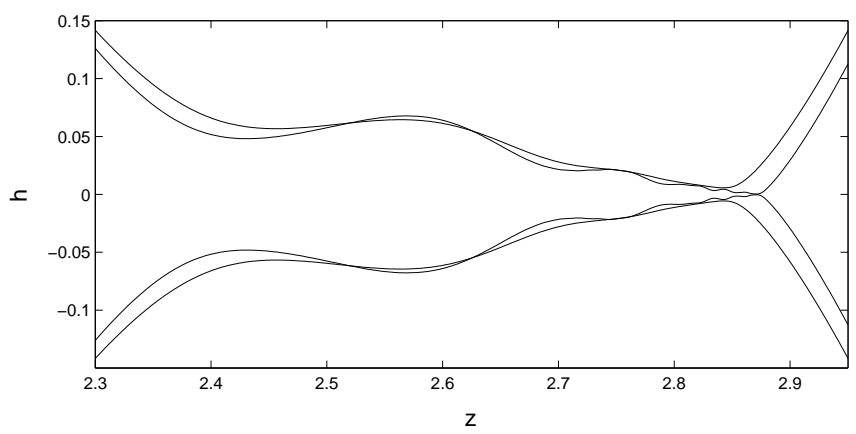

FIG. 8. Two profiles close to the singularity time. The one closer to $t_{0}$ has developed a sequence of undulations in the form of iterated structures approaching the point of breakup.

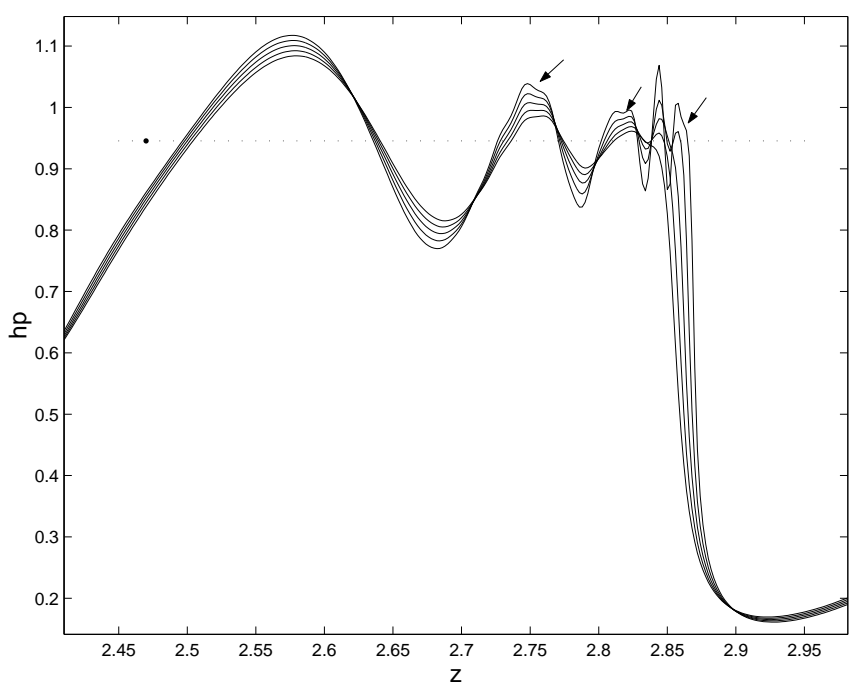

FIG. 9. The function $h p$ for various times close to $t_{0}$ where the presence of a sequence of oscillations is visible. Also, indicated with arrows, the development of secondary oscillations that will give rise to the appearance of cascades of secondary structures.

these filaments can give rise to and infinite sequence of satellite bubbles of the inner fluid inside the outer fluid. The presence of periodic structures is also visible in 9 , where we represent $h p$ which, according to the scaling laws for $h$ and $p$ near the singularity, should converge to a universal function $H P$ that tends to constant $\nu_{ \pm} \mu_{ \pm}$as $|\xi| \rightarrow \infty$. As we can see in the figure, the behaviour is not that of convergence to a constant (dotted line) but oscillatory around that constant instead. Secondary instabilities, visible in figure 9, will develop into secondary cascades of iterated filaments and hence may give rise to new cascades of subsatellite bubbles.

In order to obtain a better understanding of the origin of DSS solutions in this problem, we consider now higher order viscous effects for the pressure in (2), in the form 
(cf. [13])

$$
p=\frac{1}{h}+\frac{2}{h} \frac{\partial h}{\partial t}+\frac{\varepsilon^{2}|\log \varepsilon|}{h^{2}} \frac{\partial}{\partial z}\left(h^{4} \frac{\partial}{\partial z} \frac{1}{h}\right)
$$

(see the deduction in Supplementarial material). This leads, after the same rescaling in $z$ and $t$ leading to (3), (4), to the system formed by (4) and

$$
\frac{\partial h}{\partial t}=(p h-1)+\frac{g}{h} \frac{\partial}{\partial z}\left(h^{2} h_{z}\right)
$$

where $g \simeq 4 \lambda_{0} \varepsilon^{2}|\log \varepsilon|$. Numerically we found a transition between damped oscillations and periodic solutions (a Hopf bifurcation, as shown in the stability analysis provided in the Supplementary material) when the parameter $g \simeq 3 \cdot 10^{-4}$. A periodic orbit in self-similar coordinates implies that the breakup of the jet occurs in such a way that self-similarity holds at discrete times $t_{1}, t_{2}, \ldots$, approaching the breakup time $t_{0}$; if $T$ is the period of the orbit in selfsimilar coordinates, then $t_{N+1} / t_{N}=e^{-T}$. Then, the sequence of bubbles that appear are located at distances $d_{1}, d_{2}, \ldots, d_{N}$ from $z_{0}$ (with $d_{N} \rightarrow 0$ as $N \rightarrow \infty$ ) and will have a radius $r_{1}, r_{2}, \ldots, r_{N}, \ldots$. Using $h=e^{-\tau} H$ and $z-z_{0}=e^{-\tau} \xi$ we deduce

$$
\frac{r_{N+1}}{r_{N}}=e^{-T}, \frac{d_{N+1}}{d_{N}}=e^{-T} .
$$

Away from the Hopf bifurcations (at $g=0$, for instance) secondary instabilities may create new cascades of bubbles between consecutive bubbles which may again contain new cascades, etc, creating a fractral set. In figure 10 we represent the result, near breakup, of the evolution for initial data $h(z, 0)=1+\frac{3}{4} \cos \left(\frac{\pi z}{2}\right)$ and for $g=0$. A sequence of bubbles can be be appreciated and breakup will occur near a satellite bubble. The region around the breakup point presents the characteristic two-cone geometry plus instabilities, as in figure 8, which will give rise to new cascades of bubbles, and the process will repeat infinite times.

To summarize, we have shown the mechanism for the appearance of satellites for a viscous jet immersed into another viscous fluid with a much higher viscosity. The study of similar mechanisms for the appearance of cascade of structures (filaments) produced in very viscous jets [20] and the iterated stretching of viscoelastic filaments [21] is underway.

M.A. Fontelos is supported by the research Grant from the Spanish Ministry of Economy and Competitiveness MTM2017-89423-P.

[1] F. Savart, Mémoire sur la Constitution des Veines liquides lancées par des orifices circulaires en mince paroi, Ann. Chim. 53, 337 (1833).

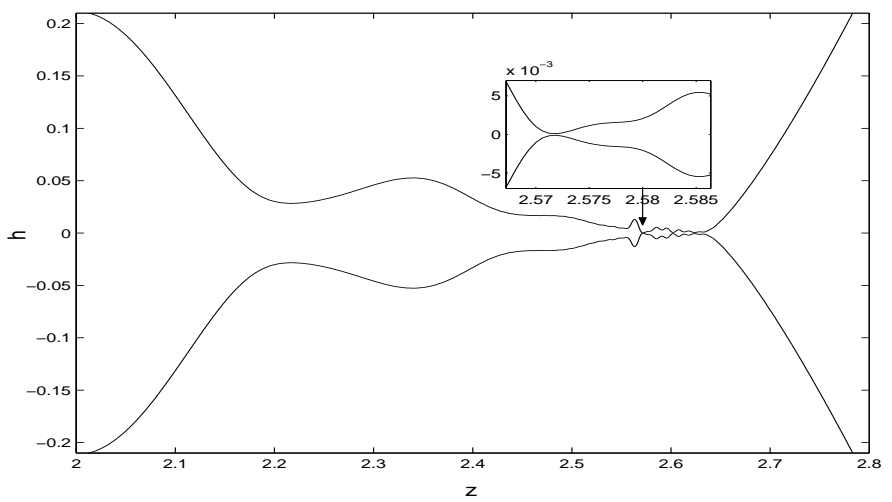

FIG. 10. Geometry of a jet near breakup for $g=0$. A sequence of bubbles can be be seen together with the detail near breakup in the neighborhood of a satellite.

[2] J. A. F. Plateau, Recherches expérimentales et théoriques sur les figures d' equilibre d'une masse liquide sans pesanteur, Acad. Sci. Brux. Mem. 23, 5 (1849).

[3] L. Rayleigh, On the instability of jets, Proc. London Math. Soc. s1-10, 4 (1879).

[4] J. Eggers and E. Villermaux, Physics of liquid jets, Rep. Prog. Phys. 71, 036601 (2008).

[5] J. Eggers, M. A. Fontelos, D. Leppinen, and J. H. Snoeijer, Phys. Rev. Lett. , 98094502 (2007).

[6] A. M. Gañán-Calvo and J. M. Gordillo, Perfectly Monodisperse Microbubbling by Capillary Flow Focusing, Phys. Rev. Lett. 87, 274501 (2001).

[7] J. M. Gordillo and M. A. Fontelos. Satellites in inviscid breakup of bubbles. Phys. Rev. Lett., 98:144503, (2007)

[8] S.T. Thoroddsen, T.G. Etoh, and K. Takehara, HighSpeed Imaging of Drops and Bubbles, Annual Review of Fluid Mechanics. Vol. 40 (2008):257-285

[9] J. Eggers, M. A. Fontelos, Singularities: Formation, Structure, and Propagation, Cambridge Texts in Applied Mathematics 2015.

[10] J. Eggers: Universal pinching of 3D axisymmetric free surface flow. Phys. Rev. Lett. 71, 3458 (1993)

[11] Michael C. Dallaston, Marco A. Fontelos, Dmitri Tseluiko, Serafim Kalliadasis, Discrete Self-Similarity in Interfacial Hydrodynamics and the Formation of Iterated Structures, Phys. Rev. Lett. 120, 034505 (2018)

[12] H. A. Stone and L. G. Leal, Relaxation and breakup of an initially extended drop in an otherwise quiescent liquid, J. Fluid Mech. 198, 399-427 (1989).

[13] M. R. Booty, D. T. Papageorgiou, M. Siegel, Q. Wang, Long-wave equations and direct simulations for the breakup of a viscous fluid thread surrounded by an immiscible viscous fluid, IMA Journal of Applied Mathematics 78, 851-867 (2013).

[14] J. C. Burton and P. Taborek, Two-dimensional inviscid pinch-off: An example of self-similarity of the second kind, Phys. Fluids 19, 102109 (2007).

[15] Sierou, A. \& Lister, J., Self-similar solutions for viscous capillary pinch-off. J. Fluid Mech., 497, 381-403 (2003).

[16] J. R. Lister and H. A. Stone, Capillary breakup of a viscous thread surrounded by another viscous fluid, Phys. Fluids 10, 2758 (1998).

[17] Q. Wang, Capillary instability of a viscous liquid thread in a cylindrical tube, Phys. Fluids 25, 112104 (2013). 
[18] F. Yang, H. A. Stone, Formation, Rupture, and Healing of an Annular Viscous Film, Phys. Rev. Let. 124, 224501 (2020).

[19] Doshi, P., Cohen, I., Zhang, W., Siegel, M., Howell, P., Basaran, O. \& Nagel, S., Persistence of memory in drop breakup: the breakdown of universality. Science, 302, 1185-1188 (2003).
[20] X. D. Shi, M. P. Brenner, and S. R. Nagel, A Cascade of Structure in a Drop Falling from a Faucet, Science 265, 219 (1994).

[21] M. S. N. Oliveira and G. H. McKinley, Iterated stretching and multiple beads-on-a-string phenomena in dilute solutions of highly extensible flexible polymers, Phys. Fluids 17, 071704 (2005). 